\title{
EGFR and Notch signaling respectively regulate proliferative activity and multiple cell lineage differentiation of Drosophila gastric stem cells
}

\author{
Chenhui Wang ${ }^{1,2}$, Xingting Guo ${ }^{1,2}$, Rongwen $\mathrm{Xi}^{1}$

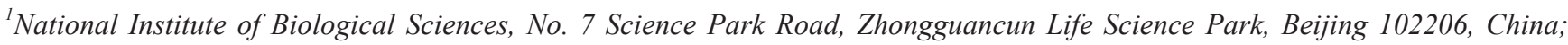 \\ ${ }^{2}$ College of Life Sciences, Beijing Normal University, Beijing 100875, China
}

Quiescent, multipotent gastric stem cells (GSSCs) in the copper cell region of adult Drosophila midgut can produce all epithelial cell lineages found in the region, including acid-secreting copper cells, interstitial cells and enteroendocrine cells, but mechanisms controlling their quiescence and the ternary lineage differentiation are unknown. By using cell ablation or damage-induced regeneration assays combined with cell lineage tracing and genetic analysis, here we demonstrate that Delta (DI)-expressing cells in the copper cell region are the authentic GSSCs that can self-renew and continuously regenerate the gastric epithelium after a sustained damage. Lineage tracing analysis reveals that the committed GSSC daughter with activated Notch will invariably differentiate into either a copper cell or an interstitial cell, but not the enteroendocrine cell lineage, and loss-of-function and gainof-function studies revealed that Notch signaling is both necessary and sufficient for copper cell/interstitial cell differentiation. We also demonstrate that elevated epidermal growth factor receptor (EGFR) signaling, which is achieved by the activation of ligand Vein from the surrounding muscle cells and ligand Spitz from progenitor cells, mediates the regenerative proliferation of GSSCs following damage. Taken together, we demonstrate that DI is a specific marker for Drosophila GSSCs, whose cell cycle status is dependent on the levels of EGFR signaling activity, and the Notch signaling has a central role in controlling cell lineage differentiation from GSSCs by separating copper/interstitial cell lineage from enteroendocrine cell lineage.

Keywords: gastric stem cell (GSSC); Notch; copper cell; interstitial cell; lineage differentiation; EGFR; Drosophila Cell Research (2014) 24:610-627. doi:10.1038/cr.2014.27; published online 7 March 2014

\section{Introduction}

Like in intestine, gastric epithelium in stomach turns over regularly and its regeneration is driven by local stem cells [1]. Dissecting out the mechanisms controlling gastric stem cell (GSSC) proliferation and differentiation should greatly facilitate our understanding of epithelial homeostasis control and gastric diseases, such as gastric cancer.

The acidic copper cell region (CCR) in the middle compartment of an adult Drosophila midgut is considered as the fly "stomach" because of the presence of

Correspondence: Rongwen Xi

E-mail: xirongwen@nibs.ac.cn

Received 26 August 2013; revised 16 December 2013; accepted 17 January 2014; published online 7 March 2014 acid-secreting copper cells (CCs) that is analogous to gastric parietal cells in mammals [2]. The recent identification of GSSCs in this region establishes a genetic system that dissects out the underlying mechanisms of stem cell regulation in stomach [3]. Drosophila GSSCs are normally quiescent, but can be promptly activated under stress conditions, such as heat shock or bacterial infection, to regenerate all types of cells found in the epithelium in copper cell region (CCR), including CCs, interstitial (IS) cells and enteroendocrine cells. The Wnt signaling is critical for the maintenance of GSSCs [3], but mechanisms controlling the quiescence and multiple cell lineage differentiation of GSSCs remain unknown. A comparative approach could be helpful, as the gastric epithelium shows a number of similarities to the bettercharacterized neighboring intestinal epithelium at the anterior (aMG) and posterior midgut (pMG): both are 
derived from a common endodermal origin and maintained by local multipotent stem cells; cell lineages derived from stem cells are also similar to a large extent but with local adoption of specific differentiation programs and cellular functions [4]. Intestinal stem cells (ISCs) in the pMG produces committed progenitors named enteroblasts (EBS), each one of which will undergo a binary fate choice to differentiate into either an absorptive enterocyte or a secretary enteroendocrine cell $[5,6]$. Notch signaling plays a central role in controlling the binary fate choice: high Notch activation promotes differentiation of an enteroblast into an enterocyte, whereas low Notch activation promotes its differentiation into an enteroendocrine cell, and the levels of Notch activation in the enteroblast is dependent on levels of the Delta (Dl) ligand produced by its mother ISC [7]. In contrast to ISC lineages in the midgut, the committed progenitor from GSSC named gastroblast (GB) appears to be subjected to a ternary fate choice to become one of the following mature cells: CC, the intermingled IS cell and enteroendocrine cell, and it is unclear whether different Notch activities could guide three distinct cellular fates. A previous study also failed to detect any Notch signaling activities in the CCR [3]. These observations raise doubt on the involvement of Notch signaling in the GSSC lineage.

Combining marker expression, cell lineage tracing and genetic analysis, here we demonstrate that D1 is a specific marker for GSSCs that sustain long-term renewal of the gastric epithelium, and Dl-Notch signaling plays a central role in guiding multiple cell lineage differentiation from GSSCs. Cell lineage tracing studies suggest that CC and IS cells are derived from a common committed progenitor whose differentiation is dependent on Notch activation, yet enteroendocrine cells are probably directly derived from GSSCs or indirectly from a separate progenitor population characterized by lack of an obvious Notch activation. We also demonstrate that stress-induced activation of epidermal growth factor receptor (EGFR) signaling, which mediates the proliferative response of ISCs [8-11], also mediates the activation of GSSCs and consequently the regeneration of the gastric epithelium. In contrast to stress conditions, the production of EGFR ligands in the CCR is kept at a minimum level under normal conditions. These observations indicate that the level of EGFR signaling activity is critical in determining the quiescence or activation status of GSSCs.

\section{Results}

Dl-Notch signaling activity in Drosophila CCR

Cellular and structural similarities between GSSC and ISC lineages prompted us to examine the potential involvement of Notch signaling in regulating GSSC lineage. First, we analyzed the expression of a Notch signaling activation reporter $\mathrm{Gbe}-\mathrm{Su}(\mathrm{H})_{\mathrm{m} 8}$-lacZ (referred to as $\mathrm{Su}(\mathrm{H})-\mathrm{lacZ}$ hereafter) in the CCR. It has been previously demonstrated that $\mathrm{Su}(\mathrm{H})$-lacZ can faithfully reflect the Notch activation in enteroblast, the committed progenitors at pMG [7]. To outline the CCR, we identified a Gal4-enhancer trap line of dve (NP3428, referred to as dve-Gal4 hereafter) which together with UAS-mcd8GFP $(\mathrm{dev}>\mathrm{GFP})$ shows virtually identical expression pattern to dve-lacZ (Figure 1A-1D), an enhancer trap marker for $\mathrm{CCs}$ and IS cells [3]. Interestingly, $\mathrm{Su}(\mathrm{H})-\mathrm{lacZ}^{+}$cells were discretely distributed in the epithelium along the length of midgut, including in the CCR (Figure 1E). Close examination revealed that $\mathrm{Su}(\mathrm{H})-\mathrm{lacZ}$ was selectively expressed in a subpopulation of diploid cells that were negative for dev > GFP. In contrast to the previous observation, we also observed the existence of Dlexpressing cells (by anti-Dl antibody and by a Dl-lacZ enhancer trap) in the CCR that were diploid, although the expression level was relatively low compared to other regions of the midgut (Figure 1E and Supplementary information, Figure $\mathrm{S} 1$ ). Notably, one $\mathrm{Dl}^{+}$cell and one $\mathrm{Su}(\mathrm{H})-\mathrm{lacZ}^{+}$cell were frequently juxtaposed to each other, forming a pair of diploid cells (Figure 1E), a pattern that is reminiscent of ISC and EB pair in pMG [7]. This observation indicates that $\mathrm{Dl}^{+}$cells could be GSSCs and $\mathrm{Su}(\mathrm{H})-\mathrm{lacZ} \mathrm{Z}^{+}$could be GBs, the committed progenitors of GSSCs. Clonal analysis showed that the newly generated $\mathrm{Dl}^{+}$cell-labeled clones frequently contain a $\mathrm{Su}(\mathrm{H})-\mathrm{lacZ}$ cell adjacent to it, further supporting that these two cells are lineage related (Supplementary information, Figure S2). To further understand their lineage relationships, we conducted several double-labeling experiments with multiple markers, including esg-Gal4, UAS-GFP (esg $>$ GFP), which marks GSSCs and GBs, and Prospero (Pros), an enteroendocrine cell marker. CCs and IS cells were readily recognizable because of their polyploidy. We found that virtually all the small diploid cells were esg $>\mathrm{GFP}^{+}$, including $\mathrm{Pros}^{+}$enteroendocrine cells, although the levels of GFP were variable among different cell types (Figure 1F). There are several pairs of markers that were mutually exclusive in any given diploid cell, including $\mathrm{Dl}$ and $\mathrm{Su}(\mathrm{H})-\mathrm{lacZ}, \mathrm{Su}(\mathrm{H})-\mathrm{lacZ}$ and Pros, Pros and Dl (Figure 1F1-F2). Within the diploid cell population (marked by esg $>$ GFP), $\sim 29 \%$ of them were $\mathrm{Dl}^{+}$ cells, $42 \%$ were Pros ${ }^{+}, 13 \%$ were $\mathrm{Su}(\mathrm{H})-\mathrm{lacZ}^{+}$and $16 \%$ were $\mathrm{Dl}^{-} \mathrm{Pros}^{-} \mathrm{Su}(\mathrm{H})$-lacZ ${ }^{-}$cells (Figure $1 \mathrm{G}$ ). Aside from the $\operatorname{Pros}^{+}$cell population that likely represents all enteroendocrine cells, the rest $58 \%$ population may represent GSSCs and committed progenitors. The heterogeneity 

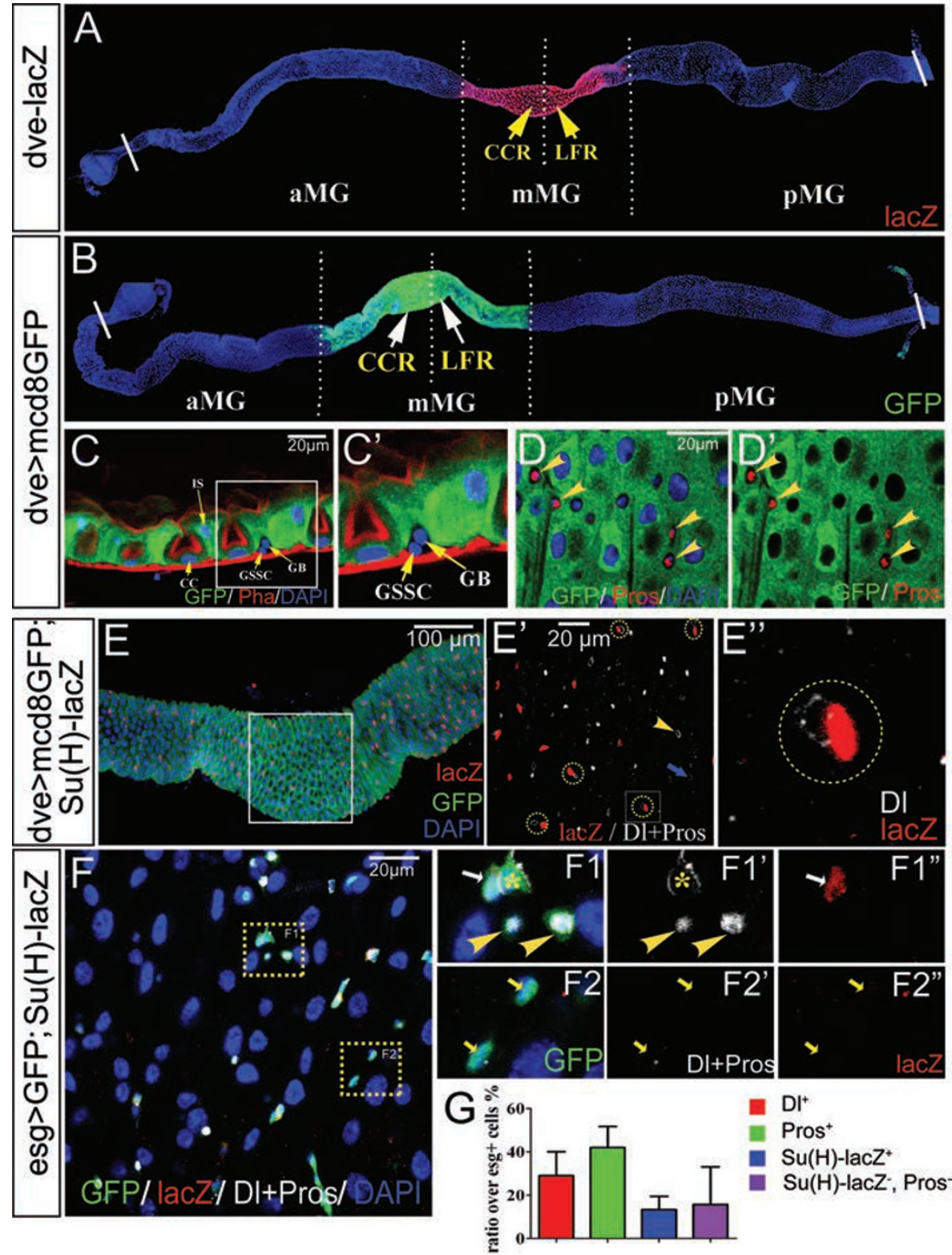

Figure 1 DI-Notch signaling activity in adult Drosophila CCR. (A) Adult Drosophila midgut can be roughly divided into three parts based on the morphology: anterior midgut (aMG), middle midgut (mMG) and posterior midgut (pMG). mMG can be marked by the expression of dve-lacZ. Copper cell region (CCR) is located at the anterior part of $\mathrm{mMG}$, and large flat cell region (LFR) is located at the posterior part of mMG. (B) The expression pattern of dve $\mathrm{NP}^{\mathrm{N} 428}$ (dve-Gal4) > mcd8GFP is similar to dve-lacZ. (CC') A saggittal section view of the CCR marked by dve-Gal4 > mcd8GFP. GFP is specifically expressed in copper cells (CC) and interstitial (IS) cells, but not in gastric stem cell (GSSC) or gastroblast (GB). CC is characterized by heavily inveginated apical surface. (D-D') A superficial view of the CCR marked by dve ${ }^{\mathrm{NP} 3428}>$ mcd8GFP (in green). Note that dve-Gal4 is not expressed in enterendocrine cells (Pros ${ }^{+}$, in red). (E) A superficial view of the CCR marked by dve-Gal4,UAS-mcd8GFP (in green) and Su(H)lacZ (in red). Su(H)-lacZ cells are present in mMG. (E') Enlarged view of the outlined region in $\mathbf{E}$, but without the display of the green channel. $\mathrm{Su}(\mathrm{H})$-lac $\mathrm{Z}^{+}$cells (in red) are frequently juxtaposed to $\mathrm{DI}^{+}$cells (in white), as indicated by dashed circles. The yellow arrowhead indicates a $\mathrm{Dl}^{\text {high }}$ cell, and the blue arrow indicates a $\mathrm{DI}{ }^{\text {low }}$ cell. (E') An enlarged view of the outlined region in $\mathrm{E}^{\prime}$ showing a pair of $\mathrm{DI}^{+}$and $\mathrm{Su}(\mathrm{H})$-lacZ $\mathrm{Z}^{+}$cell. (F) Co-staining of $\mathrm{DI}$ (in white, membrane) and Pros (in white, nucleus), esg > GFP (in green) and $\mathrm{Su}(\mathrm{H})$-lacZ (in red) markers in the CCR. In the CCR, esg ${ }^{+}$cells, which marks all diploid cells, can be classified into at least four cell types based on the combined expression status of DI, Su(H)-lacZ and Pros. (F1-F2) The expression of Su(H)lacZ, DI and Pros are mutually exclusive. Representative cell types: esg ${ }^{+}$cells that express DI (asterisk), Pros (arrowheads), or $\mathrm{Su}(\mathrm{H})$-lacZ (arrow in F1), or none of them (arrows in F2). (G) Quantification of the percentage of each subpopulation of esg ${ }^{+}$ cells in the CCR. ( $n=13$ guts quantified, error bar denotes SD.) 
within the progenitor cell population indicates that the GSSC lineage could be more complex than previously appreciated.

$\mathrm{Dl}^{+}$cells are induced to proliferate by heat stress or acute epithelial damage

The GSSCs are largely quiescent under normal conditions, but they can be readily induced to enter active cell cycles upon environmental challenge. Marker expression analyses have revealed that the mitotic cells are within esg $>\mathrm{GFP}^{+}$cell population [3]. Because esg $>\mathrm{GFP}^{+}$cell population is a heterogeneous population, which includes virtually all diploid cells in the CCR, it is unclear whether cell division occurs only in GSSCs or in the committed progenitor cells as well. We therefore asked whether $\mathrm{Dl}^{+}$cells are capable of cell division and whether they are multipotent. Heat shock treatment has been reported as a robust stimulus to induce GSSC proliferation [3]. We thus treated flies with heat shock at $37{ }^{\circ} \mathrm{C}$ for multiple times and analyzed the consequences. The treatment indeed induced mitosis in the CCR, as revealed by the mitotic marker phosphor-Histone 3 (PH3) (Figure 2A2D). Co-staining with dve $>$ GFP or esg $>$ GFP revealed that the mitotic cells were not CCs or IS cells, but were within esg $>\mathrm{GFP}^{+}$cell population (Figure $2 \mathrm{~A}-2 \mathrm{C}$ ), which agrees with the previous findings. Co-staining with D1 and Pros revealed that $\sim 89 \%(47 / 53)$ of mitotic cells were $\mathrm{Dl}^{+}$, and the rest (6/53) appeared with ambiguous or undetectable $\mathrm{Dl}$ expression. We never observed $\mathrm{Su}(\mathrm{H})$ lacZ $Z^{+}$or Pros $^{+}$cells that were $\mathrm{PH}^{+}$(data not shown). These data suggest that $\mathrm{Dl}^{+}$cells may represent the majority, if not all, of cells with proliferation potential in the CCR, indicating that Dl could be a specific marker for GSSCs.

To further test whether the $\mathrm{Dl}^{+}$cells are GSSCs and can regenerate gastric epithelium following damage, we developed a cell ablation-induced regeneration model. Previous studies have shown that in the Drosophila midgut, induction of apoptosis in enterocyte can effectively induce ISC regeneration [12]. Using a similar strategy, we attempted to induce rapid cell death in CCs and IS cells by conditionally expressing a potent cell death inducer, Reapr (rpr)-mts [13, 14] using Gal4-UAS and Gal80 ${ }^{\text {ts }}$ systems $[15,16]$. We generated flies carrying Dve-Gal4, UAS-mcd8GFP, Tub-Gal80 ts and UAS-rpr$m t s$. At permissive temperature, Gal4 activity is inhibited by Gal80, GFP and Rpr-mts are therefore not expressed. Indeed, similar to wild-type flies, a mitotic cell was rarely observed in these flies (Figure 2E). However, after shifting flies to restrictive temperature $\left(29^{\circ} \mathrm{C}\right)$ for just 24 $\mathrm{h}$, we observed a dramatic change in midgut morphology. The dve $>$ GFP domain of midgut, which includes CCR, was shrunk to such an extent that the remaining dve $>$ GFP domain was less than $1 / 5$ of the original length (Figure 2F). This phenotype could be fully suppressed by expressing p35 (Figure 2I), a potent caspase inhibitor $[17,18]$, suggesting that the shrinkage of the dve $>$ GFP domain is caused by cell death. Co-staining with PH3 at this time point revealed a significant increase of mitotic cells in the remaining dve $>$ GFP domain, suggesting that induction of cell death effectively triggers regeneration of the gastric epithelium. Consistent with this notion, the size of the dve $>$ GFP domain gradually expanded and eventually restored to the original size at 2 weeks after shifting back to the permissive temperature (Figure $2 \mathrm{G}$ and $2 \mathrm{H}$ ). Mitosis was also gradually ceased during the 2 -week period (Figure 2J). This cell ablation-induced regeneration assay demonstrates striking regenerative ability of the gastric epithelium, and indicates the existence of a potential memory mechanism that guides optimal regeneration to restore the original organ size.

We then examined these mitotic cells in the CCR by co-staining with $\mathrm{Dl}$ and Pros markers. Approximately $90 \%(73 / 80)$ of the mitotic cells were $\mathrm{Dl}^{+}$and none of them were $\operatorname{Pros}^{+}$(Figure 2K), suggesting that similar to heat shock treatment, acute ablation of differentiated cells also specifically triggers the activation of $\mathrm{Dl}^{+}$cells, the putative GSSCs.

Cell lineage tracing analysis reveals that $\mathrm{Dl}^{+}$cells are multipotent

To unambiguously demonstrate that $\mathrm{Dl}^{+}$cells are multipotent, we conducted a directed lineage tracing analysis of $\mathrm{Dl}^{+}$cells by generating flies carrying the following elements: Dl-Gal4, Tub-Gal80 $0^{t s}\left(D l-G a l 4^{t s}\right), U A S-f l p$ and the flp-out cassette $($ Act $<$ stop $<$ lacZ) [19]. This lineage tracing strategy allows conditional activation of flpase in $\mathrm{Dl}^{+}$cells and therefore all descendents of $\mathrm{Dl}^{+}$cells will be marked by the lacZ expression. As a positive control, we first conducted directed lineage tracing of $\mathrm{esg}^{+}$cells, a population known to contain GSSCs, with the esg-Gal4 system combined with the flp-out cassette. Under permissive temperature, lacZ expression was not observed in the CCR, but on day 7 after shifting to $29^{\circ} \mathrm{C}$, almost all $\mathrm{esg}^{+}$cells were labeled with nuclear lacZ, and virtually all lac $Z^{+}$cells were esg ${ }^{+}$, suggesting that the labeled GSSCs did not produce any new cells during the 1-week period (Figure 3B), a phenomenon that is consistent with the notion that GSSCs are largely quiescent. In order to activate GSSCs, we fed flies with dextran sulfate sodium (DSS) after shifting back to permissive temperature (Figure $3 \mathrm{~A}$ ). DSS is a barrier-damaging agent that has been shown to be effective in inducing ISC proliferation at pMG [20]. DSS feeding also triggered robust regenera- 


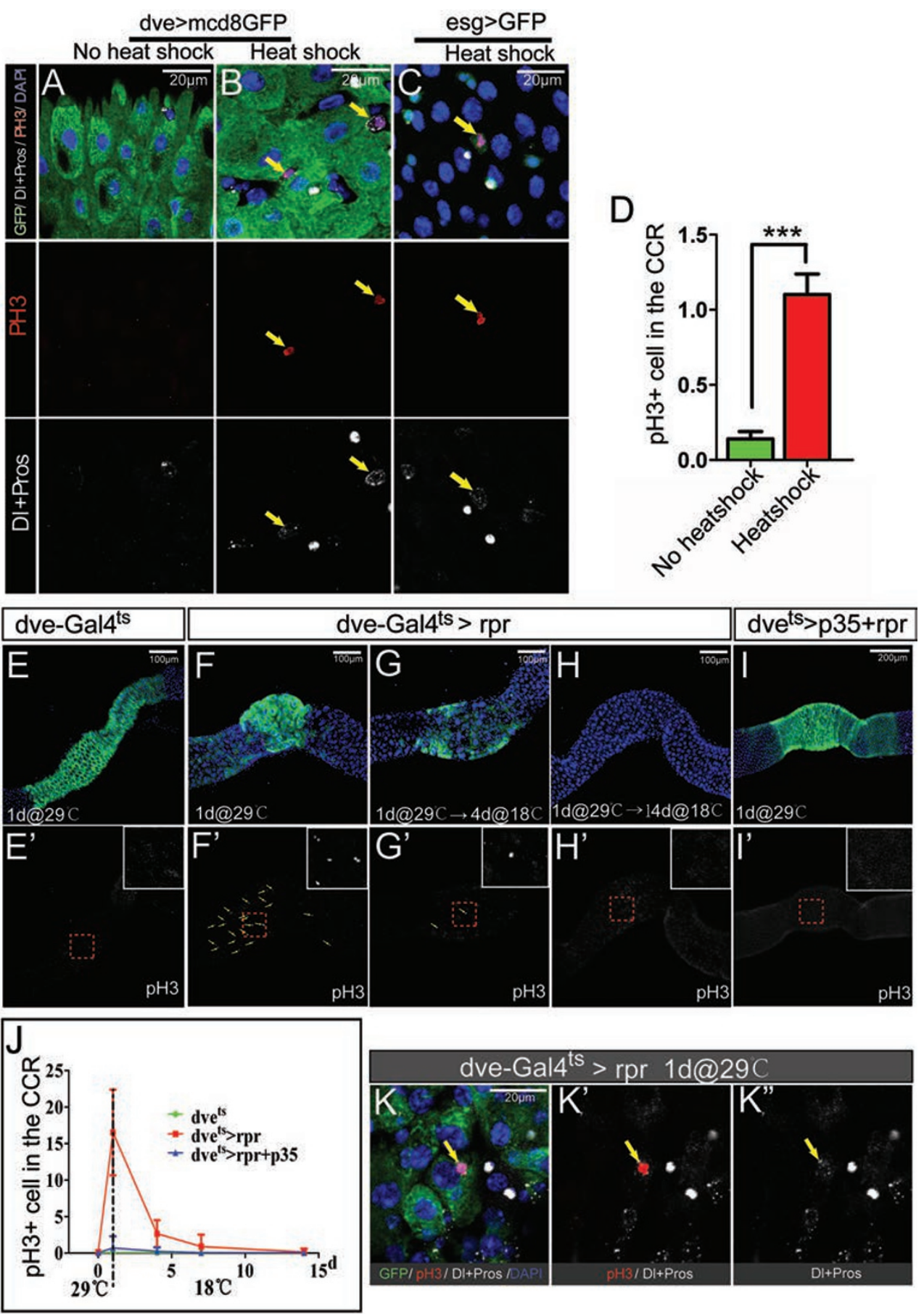

Figure $2 \mathrm{DI}^{+}$cells can be induced into mitosis by heat stress and damage. (A) Without heat shock, mitotic cell is rarely observed in the CCR. (B) The induced mitotic cells (marked by $\mathrm{PH} 3$, in red, and indicated by arrows) after heat shock treatment express DI (in white, membrane), but not dve > mcd8GFP (in green). (C) The induced mitotic cells are within esg $>$ GFP ${ }^{+}$cell population. (D) Quantification of $\mathrm{PH}^{+}$cells in the CCR. Data are represented as mean $\pm \mathrm{SD}$ (error bar). ${ }^{* *} P<0.001$. (E-F) Expression of Rpr-mts driven by dve-Gal $4^{\text {ts }}(\mathbf{F})$ for 1 day at $29{ }^{\circ} \mathrm{C}$ led to severe shrinkage of CCR and a dramatic increase of mitotic cells in the CCR (PH3, in white, and indicated by arrows). Control in E. (G-H) Recovery after genetic ablation of CCs and IS cells. The gastric epithelium was gradually restored and mitosis gradually ceased from day 4 (G, G') to day 14 (H, H') after shifting back to $18{ }^{\circ} \mathrm{C}$. (I) Expression of anti-apoptotic protein p35 completely suppressed rpr-mts-induced phenotypes. (J) Quantification of mitoses in the CCR during the cell ablation and recovery process. For each time point, 18-29 guts were quantified. Error bars denote SD. (K-K") The induced mitotic cells (PH3 ${ }^{+}$, in red, and indicated by arrow) were positive for DI (in white, indicated by arrow in $\left.\mathbf{K}^{\prime \prime}\right)$. 
A label@29"
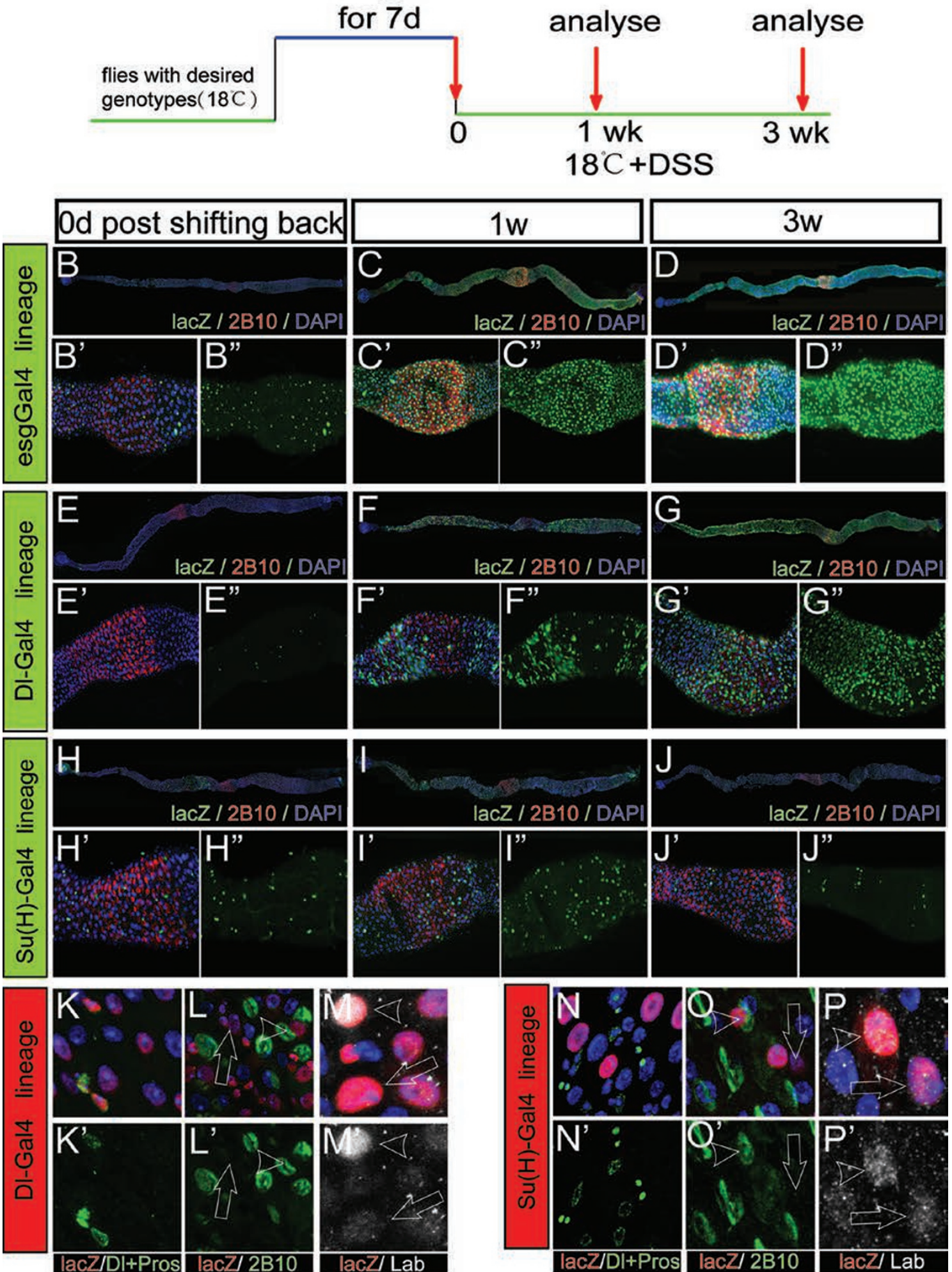
tion of gastric epithelium, evidenced by the induction of mitosis and the wide-spread expression of lacZ in virtually all cells in the CCR a week after shifting to permissive temperature (Figure 3C).

In Dl-Gal4 ${ }^{\text {ts }}$-directed lineage tracing system, $\sim 35 \%$ and $55 \% \mathrm{Dl}^{+}$cells could be labeled with nuclear lacZ in the CCR and pMG, respectively (Figure 3E and Supplementary information, Figure S3). Incomplete labeling of all $\mathrm{Dl}^{+}$cells may indicate that Dl-Gal4 is a relatively weak driver. After DSS treatment, lac $Z^{+}$cells spread out significantly in a clonal fashion, suggesting that each lac $\mathrm{Z}^{+}$cell cluster is derived from a common stem cell origin (Figure 3F). Each lacZ $\mathrm{Z}^{+}$cell cluster contains multiple cell types, including $\mathrm{Dl}^{+}$cells, enteroendocrine cells (Figure $3 \mathrm{~K}$ ), as well as CCs revealed by a monoclonal antibody 2B10 (anti-Cut) (Figure 3L), which specifically marks the inveginated apical membrane of CCs [3]. The polyploid cells derived from a common $\mathrm{Dl}^{+}$cell also showed either high or low levels of Labial (Lab) expression (Figure 3M), which indicate CCs or IS cells, respectively [3]. This cell lineage tracing experiment demonstrates that $\mathrm{Dl}^{+}$cells are multipotent and can produce all three types of differentiated cells in the CCR.

\section{$\mathrm{Dl}^{+}$cells are self-renewing GSSCs that can sustain re- petitive epithelial regeneration}

Although $\mathrm{Dl}^{+}$are multipotent, it remains possible that they could be committed progenitors with a limited proliferation capacity. If this is the case, the initially labeled cells and their descendants would be gradually diluted and disappeared from the epithelium after rounds of epithelial regeneration. We therefore performed a longterm lineage tracing experiment, which was illustrated in Figure 3A. Briefly, after labeling, flies were challenged with continuous DSS treatment for a maximum of 3 weeks before analysis. As expected, in esg-Gal4 ${ }^{\text {ts }}$ directed lineage tracing experiment, lac $\mathrm{Z}^{+}$cells remained to occupy the majority of epithelial cells in the CCR after 3 weeks of repetitive regeneration (Figure 3D). Similarly, in Dl-Gal4 $4^{\text {ts }}$-directed lineage tracing experiment, patches of lac $Z^{+}$cells were still observed in CCR (Figure $3 \mathrm{G})$. These lacZ $Z^{+}$cells include $\mathrm{Dl}^{+}$cells, Pros $^{+}$cells, and $\mathrm{Lab}^{\text {high }} \mathrm{CCs}$ and Lab ${ }^{\text {low }}$ IS cells (data not shown), suggesting that the $\mathrm{Dl}^{+}$cells remain multipotent after rounds of regeneration. These sets of lineage tracing studies demonstrate that the $\mathrm{Dl}^{+}$cells in CCR can self-renew and sustain repetitive turnover of gastric epithelium, and are therefore authentic GSSCs.

\section{$\mathrm{Su}(\mathrm{H})$-lacZ $\mathrm{Z}^{+}$cells are committed precursors of CCs and IS cells}

The identification of $\mathrm{Dl}^{+}$cells as GSSCs indicates that the $\mathrm{Su}(\mathrm{H})-\mathrm{lacZ} \mathrm{Z}^{+}$cells could be GBs, the immediate daughters of GSSCs that have committed to differentiate. To determine the multipotency of $\mathrm{Su}(\mathrm{H})$-lac $\mathrm{Z}^{+}$cells, we conducted directed lineage analysis with the $\mathrm{Su}(\mathrm{H})-\mathrm{Gal} 4^{\text {ts }}$ system. As shown in Figure $3 \mathrm{H}$, virtually all initially labeled lac $\mathrm{Z}^{+}$cells were diploid cells and were sporadically distributed in the gastric epithelium. One week after DSS treatment, these lac $Z^{+}$cells did not grow in a clonal fash-

Figure 3 Cell lineage tracing analysis demonstrates that $\mathrm{DI}^{+}$cells are multipotent and can sustain epithelial regeneration after repetitive damage. (A) Strategy for lineage tracing studies. To perform DI-Gal $4^{\text {ts }}$-, Su(H)-Gal $4^{\text {ts }}-$ and esg-Gal $4^{\text {ts }}$-directed cell lineage tracing, flies of appropriate genotypes were raised at $18{ }^{\circ} \mathrm{C}$ and shifted to $29{ }^{\circ} \mathrm{C}$ for 7 days. Flies were subsequently shifted back to $18{ }^{\circ} \mathrm{C}$ and were fed with DSS. Guts were dissected out and examined at 0 day, 1 week and 3 weeks, respectively, after shifting back to $18^{\circ} \mathrm{C}$. (B-D) esg-Gal4 $4^{\text {ts }}$-directed cell lineage tracing. (B-B") After labeling, almost all diploid cells in the CCR (marked by 2B10 staining, in red, also in C-J) were labeled with lacZ (in green, also in C-J). (C-C") After DSS treatment for 1 week, the gastric epithelium was almost completely replaced by lacZ ${ }^{+}$cells. (D-D") After DSS treatment for 3 weeks, the gastric epithelium was completely occupied by the cells derived from esg ${ }^{+}$cells. (E-G) DI-Gal $4^{\text {ts }}$-directed cell lineage tracing. (E-E') After labeling, only a fraction of diploid cells were labeled with lacZ. (F-F") After DSS treatment for 1 week, large patches of lacZ $Z^{+}$cells were found in the gastric epithelium. (G-G') The descendents of DI cells occupied most of the CCR after DSS treatment for 3 weeks. (H-J) Su(H)-Gal4 $4^{\text {ts }}$-directed cell lineage tracing. (H-H') After labeling, a fraction of diploid cells were labeled with lacZ in the CCR. (I-I") After DSS treatment for 1 week, lacZ ${ }^{+}$cells did not expand and were still sparsely distributed in the gastric epithelium. Most lacZ $Z^{+}$cells became polyploid. (J-J") After DSS treatment for 3 weeks, most lac $Z^{+}$cells disappeared from the epithelium. (K-M') All types of cells in the gastric epithelium were present in the progeny of $\mathrm{DI}^{+}$cells. (K-K') Co-staining with lacZ (in red, same as in L-P) and DI/Prospero (in green, also in N-N') revealed that $\mathrm{DI}^{+}$cells could produce enteroendocrine cells. (L-L') Costaining with lacZ and 2B10 antibody (in green, same in O-O') revealed that $\mathrm{DI}^{+}$cells could produce polyploid cells positive for 2B10 (indicated by white arrowhead), as well as polyploidy cells negative for 2B10 (indicated by white arrow), indicating that $\mathrm{DI}^{+}$cells could produce both $\mathrm{CC}$ cells and IS cells. (M-M') Co-staining with lacZ and Lab (in white, also in P-P' ) revealed that $\mathrm{DI}^{+}$cells could produce Lab ${ }^{\text {high }} \mathrm{CC}$ (indicated by white arrowhead) as well as Lab ${ }^{\text {low }}$ IS cell (indicated by white arrow). (N-P') Su(H)-Gal4 $4^{+}$cells could produce CC cells and IS cells, but not enteroendocrine cells. (N-N') The descendents of $\mathrm{Su}(\mathrm{H})-\mathrm{Gal} 4^{+}$cells were negative for both $\mathrm{DI}$ and Pros. (O-O') Both $2 \mathrm{~B}^{\prime} 10^{+}$and $2 \mathrm{~B}^{\prime} 10^{-}$ polyploidy cells were present in the descendents of $\mathrm{Su}(\mathrm{H})-\mathrm{Gal} 4^{+}$cells. (P-P') Both Lab ${ }^{\text {high }} \mathrm{CC}$ cell and Lab ${ }^{\text {low }}$ IS cell were present in the descendents of $\mathrm{Su}(\mathrm{H})-\mathrm{Gal} 4^{+}$cells. 
ion, but instead most of them became polyploid cells that were sparsely distributed (Figure 3I). Co-staining with $2 \mathrm{~B} 10$ or Lab indicates that these cells were either CCs or IS cells (Figure $3 \mathrm{~N}-3 \mathrm{P}$ ), suggesting that $\mathrm{Su}(\mathrm{H})-\mathrm{lacZ}$ cells give rise to CCs or IS cells, but not enteroendocrine cells. After repetitive damage and regeneration, the lac $Z^{+}$ were largely lost from the epithelium (Figure 3J), further suggesting that $\mathrm{Su}(\mathrm{H})-\mathrm{lacZ}^{+}$cells are post-mitotic, committed progenitors of CC/IS cells in the CCR.

\section{The EGFR/MAPK signaling mediates damage-induced GSSC activation}

Multiple studies on pMG have demonstrated an essential role of EGFR signaling in regulating ISC proliferation and mediating damage-induced ISC activation [8-11]. In response to epithelial damage or infection, ISCs have elevated EGFR signaling, which is triggered by Spitz (Spi) produced by the progenitor cells and Vein (Vn) produced by visceral muscle cells, and EGFR signaling activation is sufficient to promote ISC proliferation. To test whether EGFR also mediates the activation of GSSCs following damage, we first examined the expression of Vn by a lacZ trap line vn-lacZ, and the expression of Spi by a Gal4 trap line Spi-Gal4 with the UAS-GFP reporter (Spi > GFP). Vn-lacZ was specifically detected in visceral muscle cells along the length of midgut, except the CCR region, where its expression was barely detectable (Figure 4A, and inset). Interestingly, vn-lacZ in the CCR was promptly induced following rpr-induced ablation of CCs and IS cells (Figure 4B, and inset). Spi $>$ GFP (visualized by anti-GFP staining) was detectable in epithelial progenitor cells along the length of midgut, but was similarly downregulated in the CCR (Figure 4C). Co-staining with 2B10, anti-Dl and anti-Pros markers revealed that Spi $>$ GFP was expressed in some diploid cells in the $\mathrm{CCR}$, including $\mathrm{Dl}^{+}$GSSCs and Pros ${ }^{+}$enteroendocrine cells, and was not expressed in CCs or IS cells (Figure 4D, and data not shown). Similarly, DSS treatment also promptly induced expression of Spi $>$ GFP in the CCR (Figure 4E).

Real-time quantitative PCR (RT-qPCR) analyses for DSS-treated CCR or rpr-induced CCR revealed that in addition to Vn and Spi, the expression of another EGFR ligand, Krn, as well as two known target genes, sprouty (sty) and argos, were also promptly upregulated following damage (Figure 4F). Moreover, pErK, a direct readout for MAPK signaling activation, was significantly upregulated in the CCR following damage (Figure 4G4I). Normally, its expression could be detected in some diploid cells in the CCR (Figure 4G). Following damage, not only its expression level was significantly upregulated, but also its expression pattern was altered, as many polyploid cells displayed pErK expression (Figure 4H and 4I). Taken together, these observations demonstrate that EGFR signaling is promptly activated in the CCR following damage, indicating a possible role for EGFR/ MAPK signaling in the regeneration process.

We next determined the requirement of EGFR signaling in damage-induced GSSC activation. Expressing a dominant negative form of EGFR by esg-GAL4 significantly suppressed the proliferative response of GSSCs following DSS treatment, as revealed by BrdU incorporation assay (Figure 5C, compared to 5A and 5B). Similarly, knocking down spi in $\mathrm{esg}^{+}$cells (Figure 5F) or $v n$ in muscle cells (Figure 5G) also significantly suppressed damage-induced proliferative response of GSSCs (Figure 5I). To test whether EGFR signaling activation is sufficient to induce GSSC activation, we conditionally expressed a secreted form of Spi in the CCR. We observed rapid appearance of mitotic figures in the CCR two days after ectopic Spi expression (Figure 5H). These data demonstrate that EGFR signaling is both necessary and sufficient for damage-induced GSSC activation. Along with the ligand expression results, we conclude that EGFR signaling induced by multiple receptor ligands mediates damage-induced GSSC activation.

EGFR signaling functions in parallel with Wingless $(W g)$ signaling in promoting GSSC activation following damage

Previous studies have suggested an important role for Wg signaling in GSSC maintenance [3]. Using the esgGAL4 ${ }^{\text {ts }}$ system, we found that transiently expressing TCF.DN, a dominant negative form of TCF that can be served as an inhibitor for $\mathrm{Wg}$ signaling [21], also effectively reduced the proliferative response of GSSCs following DSS treatment (Figure 5D), an effect largely similar to EGFR signaling inhibition (Figure 5I). To determine the epistatic relationships between EGFR and $\mathrm{Wg}$ signaling in damage-induced GSSC activation, we simultaneously inhibited both pathways by coexpressing TCF.DN and EGFR.DN, and examined the effect on GSSC proliferation following DSS treatment. As revealed by BrdU labeling assay, although expressing either inhibitor alone was effective in attenuating the proliferation response, co-expressing both inhibitors drastically enhanced the effect: the percentage of BrdUlabeled progenitor cells was at background levels, similar to that in the untreated controls (Figure 5E and 5I), showing that co-inhibition of both pathways can virtually block the proliferative response following damage. These data suggest that EGFR and Wg signaling function in parallel and cooperatively to promote GSSC activation following tissue damage. 

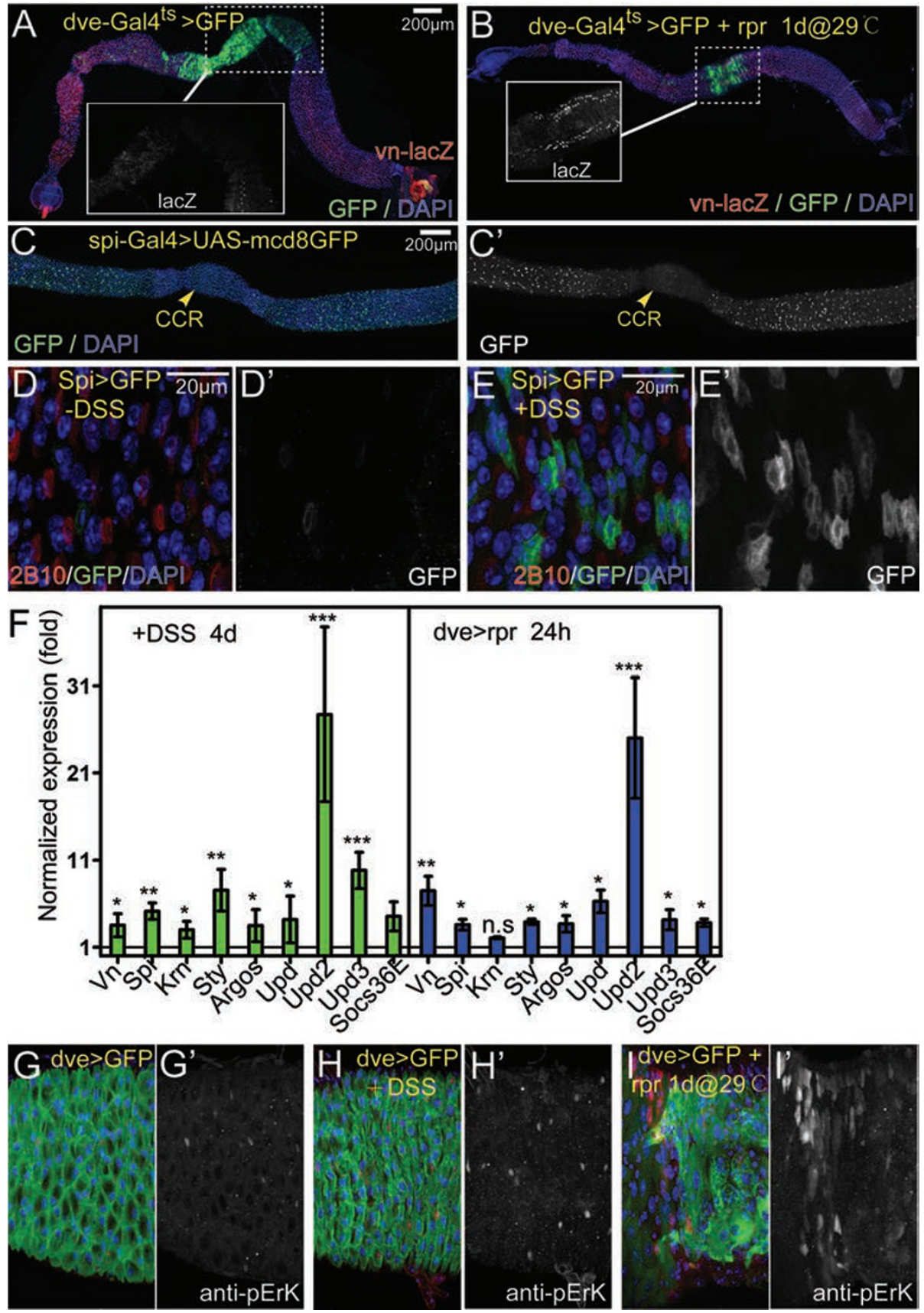

Figure 4 EGFR signaling is activated in the CCR upon damage. (A) vn-lacZ (in red) is specifically expressed in visceral muscle cells. In contrast to aMG and pMG, vn-lacZ is barely detectable in the CCR (outlined by the dashed rectangle, and the separate channel for LacZ staining was shown in inset). (B) Expression of Rpr-mts driven by dve-Gal $4^{\text {ts }}$ led to significant upregulation of vn-lacZ in muscle cells in the CCR. (C-C') The expression pattern of spi-Gal4 > UAS-mcd8GFP. GFP cells are scattered, distributed along the length of midgut, but GFP is expressed at a much lower level in the CCR compared to the levels in the aMG and pMG regions. (D-E) Expression of GFP driven by Spi-Gal4, Spi > GFP, in the CCR of control (D-D'), and DSS-treated midguts ( 7 days at $29^{\circ} \mathrm{C}$ ) (E-E'). 2B10 (in red) marks copper cells. Note that Spi $>$ GFP was significantly upregulated upon DSS treatment. (F) RT-qPCR analysis of Drosophila EGFR ligands (Vn, Spi, Krn), the transcriptional targets (sty, argos), the Jak/stat signaling ligands (upd, upd2, upd3) and the transcriptional target socs36E. Data were shown as mean \pm SD, Student's $t$-test was used to calculate the statistical significance $\left({ }^{\star} P<0.05,{ }^{* \star} P<0.01\right.$, ${ }^{* * *} P<0.001$, n.s. stands for no significant difference). (G-I) The Drosophila EGFR/MAPK signaling activity was assayed by pErK staining. (G-G') The MAPK activity in the CCR of control midgut. (H-H') MAPK activity after DSS treatment (4 days). (I-I') MAPK activity following expression of rpr with dve-Gal $4^{\text {ts }}$ at $29^{\circ} \mathrm{C}$ for $24 \mathrm{~h}$. 


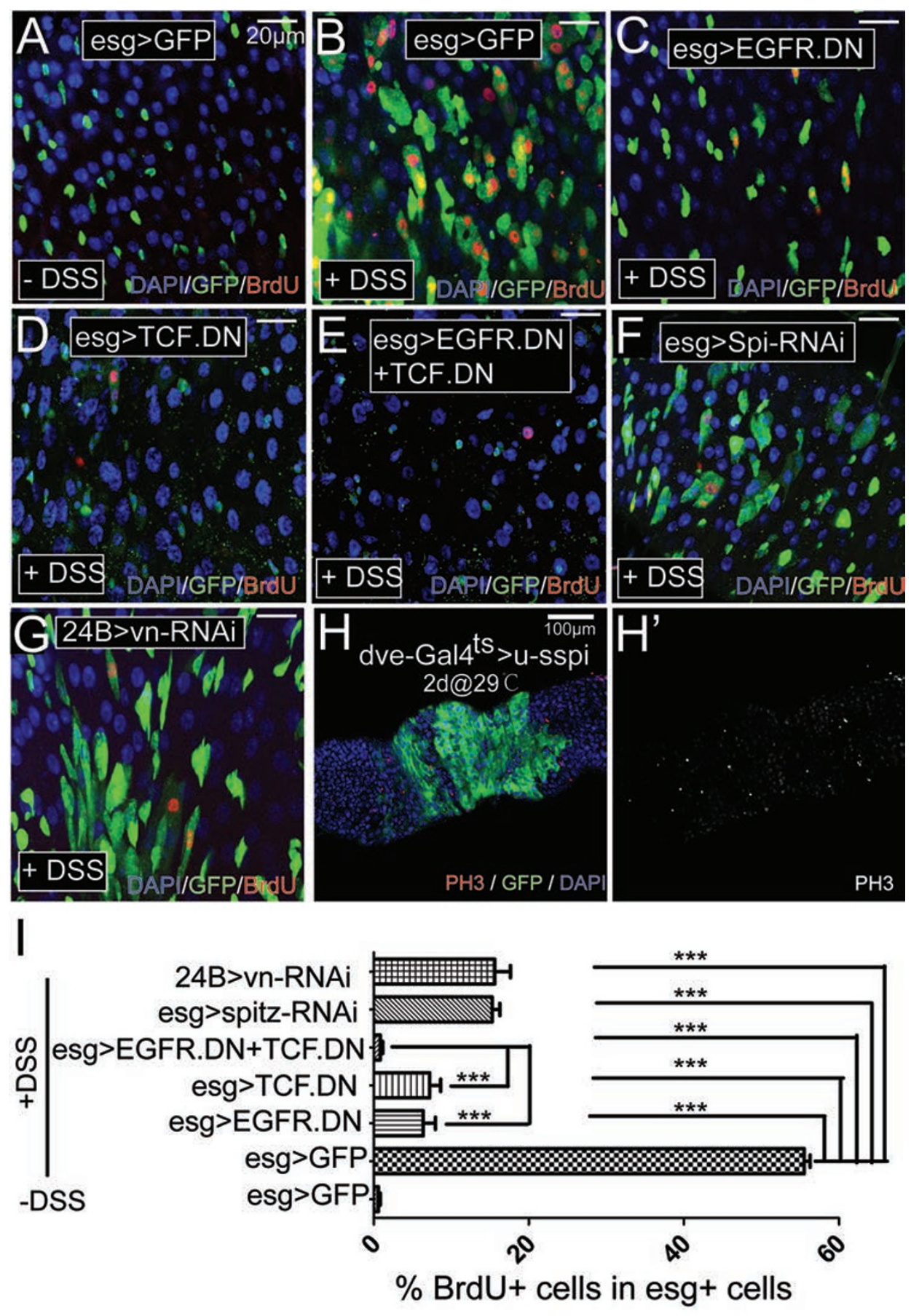

Figure 5 EGFR signaling is necessary and sufficient for damage-induced GSSC activation. (A, B) DSS treatment led to an increased $\mathrm{BrdU}$ incorporation (red) in esg > GFP (green) cells in the CCR. Mock control in A. (C) Expression of a dominant negative form of EGFR (EGFR.DN) with the esg-Gal4 driver significantly reduced BrdU incorporation following DSS treatment. (D) Expression of a dominant negative form of TCF (TCF.DN) with the esg-Gal4 driver significantly reduced BrdU incorporation following DSS treatment. (E) Simultaneous expression of EGFR.DN and TCF.DN with the esg-Gal4 driver further suppressed BrdU incorporation. (F) Expression of Spi-RNAi with the esg-Gal4 driver significantly reduced BrdU incorporation following DSS treatment. (G) Expression of vn-RNAi with the 24B-Gal4 (muscle cell) driver significantly reduced BrdU incorporation following DSS treatment. esg-GFP (green) is a GFP trap line for esg. (H-H') Ectopic expression of a secreted form of Spi (sspi) with the dve-Gal4 $4^{\text {ts }}$ driver $\left(2\right.$ days at $29^{\circ} \mathrm{C}$ ) significantly increased $\mathrm{PH}^{+}$cells (red in $\mathbf{G}$, white in $\mathbf{G}$ ') in the CCR. (I) Quantification of BrdU incorporation rate in $\mathrm{esg}^{+}$cells in the CCR. $n=10-15$ guts. Error bar denotes SD. ${ }^{* * *} P<0.001$. 
Notch signaling is required for copper/interstitial cell lineage specification

The observation that $\mathrm{Su}(\mathrm{H})-\mathrm{lac} \mathrm{Z}^{+}$cells differentiate into either CCs or IS cells but not enteroendocrine cells indicate that Notch signaling plays a role in regulating multiple cell lineage differentiation from GSSCs. To test the requirement of Notch in the GSSC lineage, we inhibited Notch activity in GSSCs and GBs by conditionally expressing Notch-RNAi with the esg-Gal4 $4^{\text {ts }}$ system. Normally, $\mathrm{Dl}^{+}$and Pros ${ }^{+}$cells are scatteredly distributed in the gastric epithelium among $\mathrm{Dve}^{+} \mathrm{CCs}$ and IS cells (Figure 6A). After shifting flies to restrictive temperature for two weeks, as expected, we observed large, tumorous cell clusters of $\mathrm{Dl}^{+}$cells and Pros cells in both aMG and pMG, because of the essential role of Notch in ISC differentiation. Interestingly, $\mathrm{Dl}^{+}$or Pros ${ }^{+}$cell clusters, although much smaller in size, were also found in the CCR (Figure 6B and 6C). To further test the function of Notch in GSSC differentiation, we also generated Notch ${ }^{55 e l l}$ (a strong loss-of-function allele of Notch) and $D l^{\text {rveF10 (a }}$ strong loss-of-function allele of $D l$ ) mutant clones using the MARCM system, and analyzed the behavior of Notch mutant GSSC clones. As a control, the wild-type GSSCs could generate clones that contained all differentiated cell types found in the CCR (Figure 6D). Notch mutant clones, however, cell-autonomously developed into clusters of small diploid cells that could be marked by either Dl or Pros, but not Lab (Figure 6E), suggesting that Notch mutant GSSCs develop into GSSC-like and enteroendocrine cell-like tumors. Similarly, $D l^{\text {rveF10 }}$ mutant GSSC clones developed into GSSC-like and enteroendocrine cell-like tumors, largely indistinguishable from Notch ${ }^{55 e l l}$ mutant clones. A subtle difference was evident though, as $D l^{r v e F I 0}$ clones frequently contained a few number of polyploid cells located at the clone margin (Figure 6F), whose differentiation is likely caused by Notch activation triggered by the neighboring wild-type cells that express Dl.

Notch activation is sufficient to induce CC/IS cell differentiation

Next, we examined the consequences of forced Notch signaling activation in progenitors by expressing Notch intracellular domain $\left(\mathrm{N}^{\mathrm{icd}}\right)$, an active form of Notch. After culturing flies of esg-Galt ${ }^{\text {ts }}$; UAS-N $N^{\text {icd }}$ at restrictive temperature for one week, we did not observe any obvious difference in the population of dve-lac $\mathrm{Z}^{+}$cells in the $\mathrm{CCR}$ between $\mathrm{N}^{\mathrm{ICD}}$-expressing and control flies (Figure 7A and 7B). The percentage of Pros ${ }^{+}$cells in the gastric epithelium remained similar as well (Figure 7A and 7C). However, the percentage of $\mathrm{esg}^{+}$cells declined slightly (Figure 7C). Notably, $\mathrm{Dl}^{+}$cells virtually disappeared in the CCR (Figure 7B and 7C). In contrast, the esg $\mathrm{Dl}^{-}$Pros ${ }^{-}$cell population, which may represent differentiating GBs, was significantly increased (Figure 7C), indicating that Notch activation may induce GSSC differentiation, and consequently the transition of $\mathrm{Dl}^{+}$cells to GBs. Because esg ${ }^{+}$cells include all diploid cells in the CCR, it remains possible that Notch activation suppresses D1 expression in GSSCs but does not affect their fate. To test this possibility, we generated heat shockinduced MARCM clones with forced $\mathrm{N}^{\mathrm{icd}}$ expression, and examined the cell fate within the clones. With this system, only GSSCs or the committed daughters of GSSCs could be labeled, which would normally develop into GSSC clones or transient clones, respectively. Strikingly, on day 7 after clone induction, all clones remaining in the CCR contained only either one or two cells. By costaining with the cell markers 2B10 and Lab, we found that virtually all one-cell clones contained either a CC or an IS cell (Figure 7D and 7G). Virtually all two-cell clones contained two polyploid cells, and in most cases, one of them was a CC and the other was an IS cell (Figure 7F), although clones containing two CCs or two IS cells were also occasionally observed (Figure 7G). These observations suggest that forced Notch activation in a GSSC or a committed progenitor randomly induces its differentiation to either a CC or an IS cell. The formation of two-cell clones is likely due to a one-time cell division event before $\mathrm{N}^{\text {icd }}$ takes effect. Taken together, the lossof-function and gain-of-function analyses demonstrate that Notch activation is both necessary and sufficient to induce CC/IS cell fate during multiple cell lineage differentiation from GSSCs.

Recent studies have revealed an important role for Decapentaplegic (Dpp) signaling in the differentiation of CCs in adult Drosophila [22, 23]. GSSCs that are mutant for Dpp signaling components, such as the receptor Thickvein (Tkv) or the downstream transcriptional factor Mothers against Dpp (Mad), can no longer effectively produce CCs $[22,23]$. To understand the epistatic relationships between Notch and Dpp signaling in CC differentiation, we asked whether forced expression of $\mathrm{N}^{\text {icd }}$ could induce differentiation of $t k v$ or mad mutant progenitor cells. Consistent with previous observations, we found that $\mathrm{CC}$ differentiation in $t k v$ or mad mutant clones was impaired, as only $\sim 17 \% t k v$ or mad mutant clones contained CCs, while $\sim 83 \%$ wild-type clones contained CCs when examined on day 14 after clone induction (Figure $7 \mathrm{M}$ ). Interestingly, although $\mathrm{N}^{\text {icd }}$ expression alone was sufficient to induce differentiation of wild-type GSSCs (Figure 7H), it failed to induce differentiation of tkv or mad mutant clones (Figure 7I-7L), as the percentage of clones containing $2 \mathrm{~B} 10^{+} \mathrm{CCs}$ remained similar to 

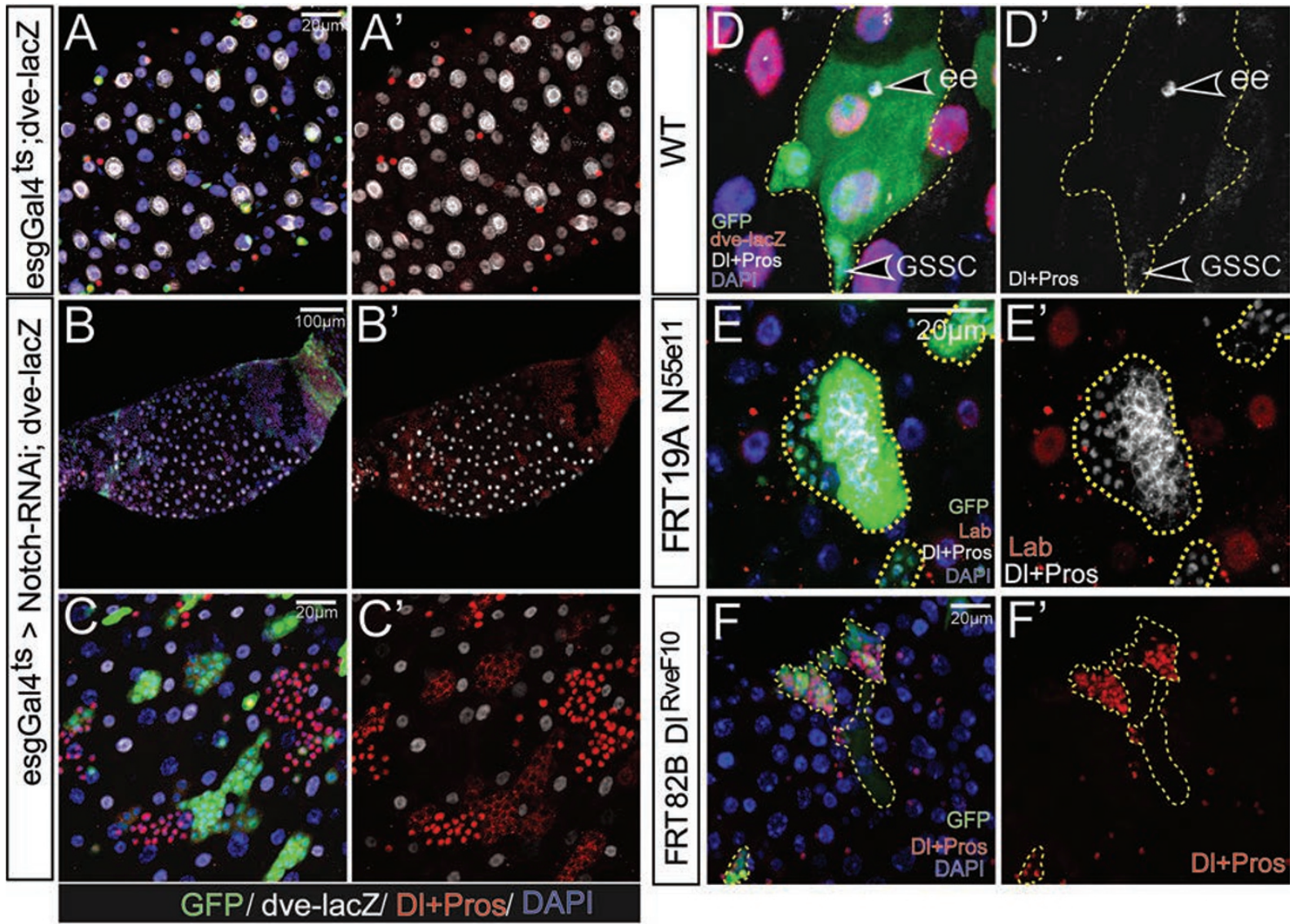

Figure 6 Notch signaling is required for CC/IS cell differentiation from GSSCs. (A-C) Midguts expressing dve-lacZ and UASGFP without (A-A') or with UAS-Notch-RNAi (B-C') driven by esgGal4 ${ }^{\text {ts }}$. Expression of UAS-Notch-RNAi led to GSSC-like (DI clusters, in red, membrane) and enteroendocrine (ee) cell-like tumors (Pros ${ }^{+}$clusters, in red, nucleus). The tumor cells were negative for dve-lacZ (in white), a marker for CC/IS cells. The GSSC-like cells are strongly marked by esg > GFP, and ee-like cells have low levels of the esg > GFP expression. (D-F) Seven-day-old GFP-labeled GSSC clones of indicated genotypes generated by the MARCM system (clones were induced by heat shock). (D-D') A wild-type clone contains one GSSC (DI', in white, indicated by arrowhead), an enteroendocrine cell (Pros ${ }^{+}$, in white, arrowhead) and several dev-lac ${ }^{+}$polyploid cells (in red). (E-E') $N^{55 e 11}$ mutant clones (GFP, in green) contain only $\mathrm{DI}^{+}$cell clusters (in white, membrane) and Pros ${ }^{+}$cell clusters (in white, nucleus), but not any Lab ${ }^{+}$cells (anti-Lab, in red). (F-F') $D I^{R v e F 10}$ mutant clones contain clusters of Pros ${ }^{+}$(in red) and Pros $^{-}$diploid cells and occasionally individual polyploid cells at the clone margin.

tkv or mad mutant clones without $\mathrm{N}^{\mathrm{icd}}$ induction (Figure $7 \mathrm{M})$. These results suggest that Dpp signaling is epistatic to Notch in regulating $\mathrm{CC}$ differentiation, indicating that Dpp signaling functions downstream of or in parallel with Notch signaling in $\mathrm{CC}$ differentiation.

\section{Discussion}

\section{$D l$ is a specific marker for Drosophila GSSCs}

One obstacle in studying tissue homeostasis is the lack of specific markers for the local stem cells. Previous studies demonstrate that esg $>\mathrm{GFP}^{+}$cells in the Drosophila gastric epithelium contains GSSCs, but esg $>$ GFP is not a stem cell-specific marker; instead, it represents virtually all diploid cells found in the region, including committed progenitors and mature enteroendocrine cells. Here we have identified Dl, the Notch ligand, as a specific marker for GSSCs. Dl is also a specific marker for midgut ISCs, suggesting certain shared properties between GSSCs and ISCs. It is not entirely surprising, as the mammalian ISCs in small intestine and GSSCs in the antrum also share a common cell surface marker Lgr5 [24]. It has been suggested that Dl expression levels in ISCs direct the fate of their differentiation-committed daughters. $\mathrm{Dl}^{\text {high }}$ ISCs induce enterocyte differentiation, whereas $\mathrm{Dl}^{\text {low }}$ ISCs induce enteroendocrine cell differentiation. We propose that a similar mechanism could be employed for GSSCs in controlling the fate choice of 

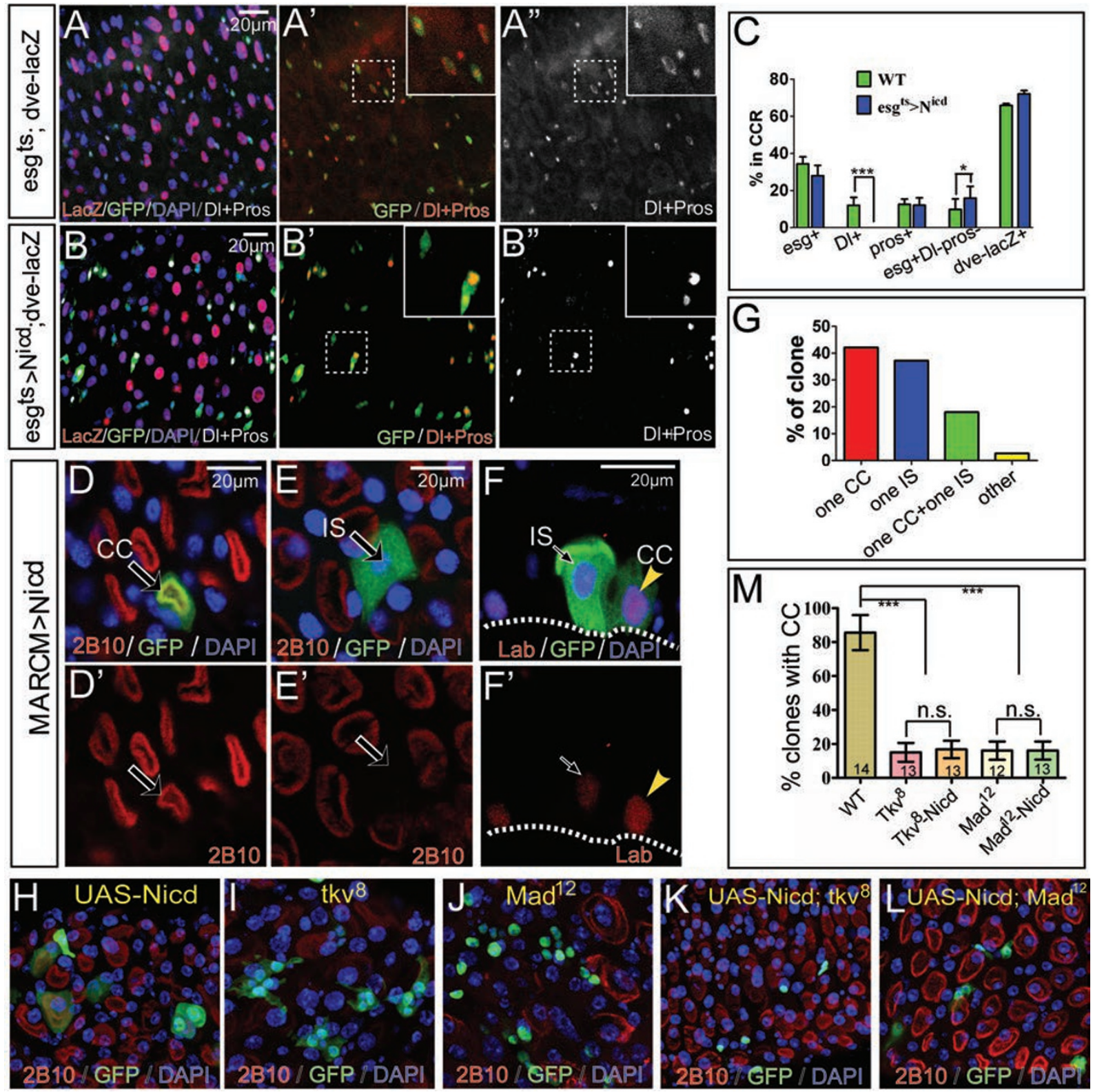

Figure 7 Forced Notch signaling activation induces differentiation of wild-type GSSCs into CC or IS cells, but not Dpp signaling mutant GSSCs. (A-C) Expression of an activate form of $N\left(N^{\text {icd }}\right)$ in the CCR by the esg-Gal4 driver for 7 days at $29^{\circ} \mathrm{C}$ led to loss of $\mathrm{DI}^{+}$GSSCs. Control in A-A", the $\mathrm{DI}^{+}$cells are no longer detected in the CCR after $\mathrm{N}^{\text {icd }}$ expression (B-B"), indicating that GSSCs are not maintained upon the $\mathrm{N}^{\text {icd }}$ expression. GFP: in green; lacZ: in red; DNA: in blue; DI \& Pros: in white (A, A", B, B') or in red (A', B'). (C) Quantification of cell types in the CCR in WT versus esg ${ }^{\text {ts }}>N^{\text {icd }}$ guts. $N=20$ guts examined, error bar denotes SD. ${ }^{* *} P<0.001,{ }^{*} P<0.05$ ( $t$-test). (D-F) $\mathrm{N}^{\text {icd }}$-expressing GFP clones in the CCR generated by the MARCM system. CC and IS cells can be distinguished by their morphology, the 2B10 marker (in red) and different expression levels of Lab (in red). (D, E) Single cell GFP clones: a clone that contains a single CC cell (D, indicated by arrow); another clone that contains a single IS cell (E, arrow). (F) Sagittal view of a GFP clone that contains one CC (indicated by arrowhead) and one IS cell (arrow) as revealed by the differential expression level of Lab (in red). Note that the CC nucleus is localized more basally compared to the IS cell nucleus. (G) Quantitative analysis of all GFP clones based on cell number and type. A total of 183 clones in the CCR from 37 guts were analyzed. (H-M) Epistatic analysis of Notch and Dpp signaling in CC specification. The MARCM system was used to generate GFP-marked clones in the CCR. Clones expressing $N^{i c d}(\mathbf{H})$, mutant for $t k v^{8}(\mathbf{I})$, mutant for $\operatorname{Mad}^{12}(\mathrm{~J}), t k v^{8}$ mutant clones co-expressing $N^{\text {icd }}(\mathrm{K})$, and $\mathrm{Mad}^{12}$ mutant clones co-expressing $\mathrm{N}^{\text {icd }}(\mathrm{L})$ were shown. In $t k v^{8}$ or $\mathrm{Mad}^{12}$ mutant GSSCs, expression of $\mathrm{N}^{\text {icd }}$ could no longer effectively induce CC differentiation. Quantitative data on the percentage of clones with CCs for various genotypes was shown in $\mathbf{M}$. Clones were examined two weeks after clone induction. Number of guts examined as indicated. Error bar denotes SD. ${ }^{* *} P<0.001$. 
their differentiation-committed daughters. Indeed, our double staining analysis with $\mathrm{Dl}$ and other markers suggest that $\mathrm{Dl}^{+}$cells probably represent most but not all GSSCs, and $\mathrm{Dl}^{-}$or $\mathrm{Dl}^{\text {low }}$ GSSCs could be included within the esg ${ }^{+} \mathrm{Dl}^{-} \mathrm{Su}(\mathrm{H})-\mathrm{lacZ}^{-} \mathrm{Pros}^{-}$cell population. Definite approval of the existence of $\mathrm{Dl}^{-}$GSSCs requires identification of specific markers for this cell population. Analysis of GSSC-derived clones has suggested that there is no unipotent GSSCs dedicated to enteroendocrine cell production (data not shown), indicating that, similar to midgut ISCs, $\mathrm{Dl}^{-}$and $\mathrm{Dl}^{+}$are two interchangeable states of a given GSSC (Supplementary information, Figure S6).

EGFR signaling regulates the cell cycle status of GSSCs

One important feature of GSSCs in the CCR is that they are largely quiescent under normal conditions but could be promptly induced to proliferate following damage. Here we have revealed an important role of EGFR signaling in mediating the GSSC activation following damage. The damage induces local expression of several EGFR ligands, including Vn, which is expressed in surrounding muscle cells, and Spi, which is expressed in GSSCs and progenitor cells, and induction of both ligands is important for EGFR signaling activation and consequently GSSC activation. Interestingly, these ligands are normally expressed in the CCR, but their expression levels are significantly lower compared to those in the aMG and pMG regions. Because ISCs at the aMG and $\mathrm{pMG}$ divide more frequently $[3,5,6]$, the expression levels of $\mathrm{Vn}$ and Spi ligands along the length of midgut seem to be largely correlated with regional stem cell activity. Based on these observations, we propose that the cell cycle status of GSSC is dependent on the levels of EGFR activity. A similar conclusion has also been drawn by a recent study [25]. Normally, GSSCs have minimum EGFR signaling activity and therefore remain largely "quiescent", as "quiescence" is probably not equal to cell cycle arrest. Consistent with this notion, knocking down Notch in esg ${ }^{+}$cells could lead to gradual accumulation of undifferentiated GSSCs over time (Figure 6B and 6C). In addition, a long-term cell lineage tracing study shows that the newly labeled GSSCs could regenerate the entire gastric epithelium in 55 days under normal conditions (Supplementary information, Figure S4). These observations suggest that GSSCs normally do divide, but at a much lower rate compared to ISCs in other regions of the midgut.

How EGFR ligands are regulated, especially following damage, remains to be investigated. In $\mathrm{pMG}$, damage or bacterial infection can induce expression of Upd-family ligands through JNK, Hippo and/or other unknown mechanisms $[10,12,26-28]$. These secreted ligands are able to promote ISC proliferation directly and also indirectly through induction of EGFR ligands [11, 29, 30]. However, the regulatory relationships among these pathways involved in regeneration are rather complex and could be distinct under different damage conditions. Given great similarities in the regulation of GSSCs and ISCs, these signaling pathways could also participate in the regeneration of gastric epithelium. Indeed, the Updfamily ligands are also upregulated following gastric injury (Figure $4 \mathrm{~F}$ ), and the JAK/STAT pathway is hyperactivated revealed by an increased expression of the $10 \times$ STAT-GFP reporter (Supplementary information, Figure S5). Thus, the gastric epithelium may be an alternative system to elucidate signaling hierarchy and cross relationships during tissue regeneration that is driven by the activation of local stem cells.

\section{Relationships between CC and IS cell in the GSSC lin- eage}

Our studies have also revealed new insights into cell hierarchy and lineage relationships in the GSSC lineage. Notch-activated cells, reflected by Notch activation reporter expression, are always adjacent to $\mathrm{Dl}^{+} \mathrm{GSSCs}$, suggesting that they are GBs. Cell lineage tracing analysis has revealed that these cells invariably adopt $\mathrm{CC}$ or IS cell fate, suggesting that they are progenitors of polyploid cell lineages. This observation indicates a separate population of GBs that are committed to enteroendocrine cell fate. This population would likely be within the esg ${ }^{+}$ $\mathrm{Dl}^{-} \mathrm{Su}(\mathrm{H})-\mathrm{lacZ} \mathrm{Z}^{-}$Pros $^{-}$cell population as well. Alternatively, enteroendocrine cells could be directly differentiated from $\mathrm{Dl}^{-}$GSSCs (Supplementary information, Figure S6). Functional analysis of Notch signaling demonstrates that Notch activity is necessary and sufficient for $\mathrm{CC} / \mathrm{IS}$ cell differentiation, which is consistent with the Notch activation pattern in the GSSC lineage. How the choice between CC/IS cell differentiation is achieved is unclear. Forced Notch activation seems to induce random GB differentiation into either CC or IS cell fate, indicating that unknown mechanisms must be involved to determine the binary fate choice. A possible factor would be $\mathrm{Lab}$, which is a homeotic transcription factor, and is differentially expressed in CCs and IS cells. During midgut development, it is required for the specification of CCs from endoderm [31, 32], and its expression is induced by Decapentaplegic and Wingless signals from the mesoderm [33]. Interestingly, Lab is also required in adult stomach for CC differentiation. However, Lab is not expressed in progenitor cells, and is therefore unlikely to be involved in the binary fate choice of GBs toward CCs or IS cells, although it is required for the function or fate 
maintenance of CCs.

Interestingly, in contrast to the adult gastric cells, embryonic gastric cells show very different patterns of Notch activation. D1 is expressed in embryonic CCs and Notch is activated only in embryonic IS cells, and this Notch activity seems to non-cell-autonomously regulate $\mathrm{CC}$ function, but not their specification [34]. Therefore, mechanisms exploited to control gastric cell specification from adult GSSCs could be very different from that controlling gastric cell specification from the endoderm during development.

$\mathrm{CC}$ is best known as the acid-secreting cell in the CCR. In contrast, little is known about the function of IS cell. Ultrastructual analysis shows that IS cells have small and loosely packed microvilli on the apical membrane, and the basal surface display extensive arrays of infoldings, or termed basal extracellular labyrinth [31, $35,36]$. These characteristics suggest an absorptive role for IS cells. CC and IS cell are alternatively aligned in the gastric epithelium, such that each CC is surrounded and enveloped by IS cells and vice versa. This pattern of organization indicates a possible regulatory/functional relationship between them. In the corpus of the mammalian stomach, the acid-secreting parietal cells are closely associated with progenitors of chief cells, whose differentiation seems to be dependent on parietal cells, as genetic ablation of parietal cells caused failure of chief cell differentiation $[37,38]$. The embryonic IS cells in Drosophila have activation of Notch signaling by receiving D1 signals from CCs, and Notch activation in IS cells is important for the absorptive function of CCs [34]. Therefore, the potential mutual communications and functional interactions between CCs and IS cells in the adult stomach are worthy of further investigation.

\section{Notch is a key regulator in the GSSC lineage}

Our data suggest that similar to the midgut stem cell lineage, Notch plays a critical role in regulating cell lineage choice in the GSSC lineage: its activation is necessary and sufficient to distinguish the CC/IS cell lineage from the enterendocrine cell lineage. One major target of Notch signaling in directing cell differentiation in midgut ISCs is the genes of the enhancer of split complex [39], and it is possible that a similar mechanism could be involved in CC/IS cell differentiation. In mammals, Notch signaling components are expressed in the gastric epithelium [40, 41], and genetic studies have suggested a requirement of Notch signaling in GSSC maintenance [42]. Notch signaling is also a major determinant in fate choice between luminal and glandular cells in chicken stomach [43]. These observations indicate that some aspects of Notch signaling in regulating GSSC differentia- tion are evolutionarily conserved.

Apart from Notch, activation mutations in the EGFR/ Ras signaling pathway are commonly found in human gastric cancers $[44,45]$. In addition, $\mathrm{Wg} / \mathrm{Wnt}$ is also known as an important maintenance factor for GSSCs and stem cells at other regions of the digestive tract, including the pMG, the hindgut and the cardia/proventriculus [3, 9, 46-49], and is known as a major self-renewal factor in mammalian intestinal and gastric stem cells [1, 24]. With the identification of EGFR and Wg signaling as critical proliferation factors and Notch signaling as a key differentiation factor for GSSCs, the Drosophila GSSC lineage should continue to serve as a powerful genetic model for further understanding of GSSC self-renewal, cell lineage specification and gastric function, which may ultimately contribute to our understanding of gastric homeostatic control and diseases in humans.

\section{Materials and Methods}

\section{Drosophila strains}

Flies were cultured on standard food media with yeast paste added to the food surface. The culture temperature was $25^{\circ} \mathrm{C}$ unless otherwise noted. Strains used in this study: dve-lacZ (gift from H Nakagoshi); dve ${ }^{\mathrm{NP} 3428} / \mathrm{CyO}$ (dve-Gal4); spi $^{\mathrm{NP} 0261}$ (spi-Gal4); Dl-Gal4 and $\mathrm{Su}(\mathrm{H})-\mathrm{Gal} 4$ (gift from S Hou) [50]; esg-Gal4, UASGFP (gift from S Hayashi); Gbe-Su(H)m8-lacZ (gift from S Bray) [51]; UAS-rpr-mts (gift from H Steller) [13]; Tkv [8], mad [12], UAS-N $^{\text {icd }}$ (gift from T Xie); UAS-EGFRDN (gift from Z Wang); UAS-s.spi (gift from T Volk) [52]; UAS-vn-RNAi (VDRC); UASspi-RNAi (NIG); Dl-lacZ (P(PZ)Dl $\left.{ }^{05151}\right)$; vn-lacZ $\left(\mathrm{vn}^{10567}\right)[53]$; UAS-lab-RNAi (BSC\#26753); $\mathrm{N}^{55 \mathrm{e} 11}$; $\mathrm{Dl}^{\mathrm{RveF10}}$; UAS-p35; UASN-RNAi; UAS-flp; UAS-mcd8GFP; Act $<$ stop $<$ lacZ and tubgal $80^{\text {ts }}$ were from Bloomington Stock Center.

\section{DSS feeding}

For DSS feeding, flies were cultured in standard food vials with regular food plus $200 \mu \mathrm{l}$ of $20 \%$ sucrose solution with $5 \%$ DSS (Sigma). The chemical solution was gently mixed with the food on the surface and left it half-dried before feeding. Flies were transferred to newly prepared vials every 2-3 days.

\section{MARCM clone induction}

GFP-labeled clones in gut epithelial cells were generated with the MARCM system [54], as previously described [9, 47, 55]. To induce clones in the gastric epithelium, 3-5-day-old female flies of appropriate genotypes were subjected to heat shock treatments in a running water bath at $37^{\circ} \mathrm{C}, 1 \mathrm{~h}$ each time, twice a day for two consecutive days. Flies were analyzed 7-14 days after the last heat shock treatment.

\footnotetext{
Dl-Gal4 ${ }^{t s}$-, Su(H)-Gal4 ${ }^{\text {ts }}$ - and esg-Gal4 ${ }^{\text {ts }}$-directed cell lineage tracing experiments

At $18{ }^{\circ} \mathrm{C}$, flies carrying Dl-Gal4 ${ }^{\text {ts }}, \mathrm{Su}(\mathrm{H})-\mathrm{Gal} 4^{\text {ts }}$ and esg-Gal4 $4^{\text {ts }}$ were crossed with flies of UAS-flp, tub-gal $80^{\text {ts }}$; Act $<$ stop $<$ lacZ, tub-gal $80^{\text {ts }}$, respectively. Three-five-day-old F1 female progeny of appropriate genotypes were subsequently shifted to $29^{\circ} \mathrm{C}$ for
} 
labeling or kept at $18{ }^{\circ} \mathrm{C}$ as controls for detecting leakiness. For all directed-cell lineage tracing experiments, three copies of TubGal80 transgenes were eventually introduced into the system, because we notice that, at permissive temperature, at least three copies of transgenes are required for complete prevention of leaky induction of flpase and consequently the lacZ expression in the gastric epithelium. After cultured at $29^{\circ} \mathrm{C}$ for 7 consecutive days, flies were then shifted back to permissive temperature, and were treated with DSS feeding as described above. Flies were then dissected and analyzed 1 week or 3 weeks after shifting back to $18{ }^{\circ} \mathrm{C}$.

\section{BrdU incorporation assay}

For BrdU labeling shown in Figure 7, adult flies were cultured at $29{ }^{\circ} \mathrm{C}$ for 7 consecutive days with or without DSS treatment. Subsequently, the flies were reared on regular food with $200 \mu \mathrm{lof} 5 \mathrm{mg} /$ $\mathrm{ml} \mathrm{BrdU}$ (Sigma) plus $20 \%$ sucrose at $29{ }^{\circ} \mathrm{C}$ for $3 \mathrm{~h}$. Midguts were then dissected and further processed as previously described [56].

\section{$R T-q P C R$}

RNA was extracted by TRIzol (Invitrogen) from $\sim 100$ middle midguts (aMG and pMGs were removed) for each sample. cDNAs were synthesized using high fidelity cDNA Synthesis Kit (Roche). RT-qPCR was performed using SYBR PrimeScript RT-PCR kit (Takara) on an ABI PRISM 7500 fast Real-time PCR System (Applied Biosystems). RT-qPCR was performed in duplicate on each of three independent biological replicates. GAPDH was used as a normalization control. Primers sequences were shown below: GAPDH forward: 5'-GAAATTAAGGCCAAGGTTCAGG-3'; GAPDH reverse: 5'-GTACCAAGAGATCAGCTTC-3' Vn forward: 5'-GTGAAGTTGCCTGGATTCGT-3' Vn reverse: 5'-CTACAGGGAGCGACTGATGC-3' Spi forward: 5'-TACCAGGCATCGAAGGTTTC-3' Spi reverse: 5'-GACCCAGGCTCCAGTCACTA-3' Krn forward: 5'-CGAGCCATCAATCTCCTTGT-3' Krn reverse: 5'-AACGATGGCACCTGCTTTAC-3' Sprouty forward: 5'-CACTACCAGAACGCGCTAAAC-3' Sprouty reverse: 5'-AAGCGATTGGTTGGTTGGCT-3' Argos forward: 5'-CCGGTGCATAAGTTGCCAGT-3' Argos reverse: 5'-GCTTTCGCACCGTGAACAAT-3' Upd forward: 5'-CCACGTAAGTTTGCATGTTG-3' Upd reverse: 5'-CTAAACAGTAGCCAGGACTC-3' Upd2 forward: 5'-TAGAGGACCACCCGACCAAT-3' Upd2 reverse: 5'-TTGGCTTGGTTTGGCAACTG-3' Upd3 forward: 5'-GAGCACCAAGACTCTGGACA-3' Upd3 reverse: 5'-CCAGTGCAACTTGATGTTGC-3' Socs36E forward: 5'-CAGTCAGCAATATGTTGTCG-3' Socs36E reverse: 5'-ACTTGCAGCATCGTCGCTTC-3'

\section{Immunostaining and microscopy}

Drosophila midguts were dissected and immunostained as described previously [47]. For staining with 2B10 (anti-Cut, Developmental Studies Hybridoma Bank (DSHB), used in 1:20 dilution), the above protocol was modified as follow: the midguts were fixed in $4 \%$ formaldehyde (electron microscopy grade) for $30 \mathrm{~min}$, and subsequently washed by $1 \times$ PBT three times, with $30 \mathrm{~min}$ each time. We find that fixation without dehydration by methanol is critical for the visualization of apical membrane staining on copper cells. Because Cut is a nuclear transcription factor and is not expressed in midgut, the staining pattern likely represents an unknown epitope that can be recognized by this antiserum. Other antisera and dyes used in this study: mouse anti-Dl (DSHB, 1:100); mouse anti-Pros (DSHB, 1:300); rabbit anti-Labial (gift from TC Kaufman ); rat anti-BrdU (Abcam, 1:300); rabbit anti-phosphoHistone H3 (Upstate, 1:1 000); rabbit polyclonal anti- $\beta$-gal (Cappel, 1:6 000); mouse anti- $\beta$-gal (DSHB, 1:50); rabbit anti-GFP (Invitrogen, 1:1 000); rabbit anti-pErK (Cell signaling, 1:200). Secondary antibodies, including goat anti-rabbit, anti-mouse, or anti-rat IgGs, conjugated to Alexa $(488,568$, or Cy5) (Molecular Probes) were used at a dilution of 1:300; rhodamine-conjugated Phalloidin (Molecular Probes, 1:500); DAPI (49,69-diamidino-2-phenylindole, Sigma; $0.1 \mathrm{mg} / \mathrm{ml}, 5 \mathrm{~min}$ incubation). Images were captured by either a Zeiss Imager Z1 equipped with an ApoTome system or a Zeiss Meta 510 confocal microscope. All images were processed in Adobe Photoshop and Illustrator.

\section{Acknowledgments}

We thank the fly community for generously providing fly stocks and antibodies, and the Bloomington Stock Center, Vienna Drosophila RNAi Center (VDRC), National Institute of Genetics (NIG) and Developmental Studies Hybridoma Bank (DSHB) for reagents. This work was supported by the Ministry of Science and Technology of China (973 program; 2011CB812700) to RX.

\section{References}

1 Schepers A, Clevers H. Wnt signaling, stem cells, and cancer of the gastrointestinal tract. Cold Spring Harb Perspect Biol 2012; 4:a007989.

2 Dubreuil RR. Copper cells and stomach acid secretion in the Drosophila midgut. Int J Biochem Cell Biol 2004; 36:745752.

3 Strand M, Micchelli CA. Quiescent gastric stem cells maintain the adult Drosophila stomach. Proc Natl Acad Sci USA 2011; 108:17696-17701.

4 Takashima S, Hartenstein V. Genetic control of intestinal stem cell specification and development: a comparative view. Stem Cell Rev 2012; 8:597-608.

5 Ohlstein B, Spradling A. The adult Drosophila posterior midgut is maintained by pluripotent stem cells. Nature 2006; 439:470-474.

6 Micchelli CA, Perrimon N. Evidence that stem cells reside in the adult Drosophila midgut epithelium. Nature 2006; 439:475-479.

7 Ohlstein B, Spradling A. Multipotent Drosophila intestinal stem cells specify daughter cell fates by differential notch signaling. Science 2007; 315:988-992.

8 Jiang H, Grenley MO, Bravo MJ, Blumhagen RZ, Edgar BA. EGFR/Ras/MAPK signaling mediates adult midgut epithelial homeostasis and regeneration in Drosophila. Cell Stem Cell 2011; 8:84-95.

9 Xu N, Wang SQ, Tan D, Gao Y, Lin G, Xi R. EGFR, Wingless and JAK/STAT signaling cooperatively maintain Drosophila intestinal stem cells. Dev Biol 2011; 354:31-43.

10 Biteau B, Jasper H. EGF signaling regulates the proliferation of intestinal stem cells in Drosophila. Development 2011; 138:1045-1055.

11 Buchon N, Broderick NA, Kuraishi T, Lemaitre B. Drosophila 
EGFR pathway coordinates stem cell proliferation and gut remodeling following infection. BMC Biol 2010; 8:152.

12 Jiang H, Patel PH, Kohlmaier A, Grenley MO, McEwen DG, Edgar BA. Cytokine/Jak/Stat signaling mediates regeneration and homeostasis in the Drosophila midgut. Cell 2009; 137:1343-1355.

13 Sandu C, Ryoo HD, Steller H. Drosophila IAP antagonists form multimeric complexes to promote cell death. J Cell Biol 2010; 190:1039-1052.

14 Lin G, Zhang X, Ren J, et al. Integrin signaling is required for maintenance and proliferation of intestinal stem cells in Drosophila. Dev Biol 2013; 377:177-187.

15 Brand AH, Perrimon N. Targeted gene expression as a means of altering cell fates and generating dominant phenotypes. Development 1993 ; 118:401-415.

16 McGuire SE, Mao Z, Davis RL. Spatiotemporal gene expression targeting with the TARGET and gene-switch systems in Drosophila. Sci STKE 2004; 2004:p16.

17 Hay BA, Wolff T, Rubin GM. Expression of baculovirus P35 prevents cell death in Drosophila. Development 1994; 120:2121-2129.

18 Clem RJ, Fechheimer M, Miller LK. Prevention of apoptosis by a baculovirus gene during infection of insect cells. Science 1991; 254:1388-1390.

19 Struhl G, Basler K. Organizing activity of wingless protein in Drosophila. Cell 1993; 72:527-540.

20 Amcheslavsky A, Jiang J, Ip YT. Tissue damage-induced intestinal stem cell division in Drosophila. Cell Stem Cell 2009; 4:49-61.

21 van de Wetering M, Cavallo R, Dooijes D, et al. Armadillo coactivates transcription driven by the product of the Drosophila segment polarity gene dTCF. Cell 1997; 88:789-799.

22 Guo Z, Driver I, Ohlstein B. Injury-induced BMP signaling negatively regulates Drosophila midgut homeostasis. J Cell Biol 2013; 201:945-961.

23 Li H, Qi Y, Jasper H. Dpp signaling determines regional stem cell identity in the regenerating adult Drosophila gastrointestinal tract. Cell Rep 2013; 4:10-18.

24 Barker N, Huch M, Kujala P, et al. Lgr5(+ve) stem cells drive self-renewal in the stomach and build long-lived gastric units in vitro. Cell Stem Cell 2010; 6:25-36.

25 Strand M, Micchelli CA. Regional control of Drosophila gut stem cell proliferation: EGF establishes GSSC proliferative set point \& controls emergence from quiescence. PLoS One 2013; 8:e80608.

26 Ren F, Wang B, Yue T, Yun EY, Ip YT, Jiang J. Hippo signaling regulates Drosophila intestine stem cell proliferation through multiple pathways. Proc Natl Acad Sci USA 2010; 107:21064-21069.

27 Karpowicz P, Perez J, Perrimon N. The Hippo tumor suppressor pathway regulates intestinal stem cell regeneration. Development 2010; 137:4135-4145.

28 Buchon N, Broderick NA, Chakrabarti S, Lemaitre B. Invasive and indigenous microbiota impact intestinal stem cell activity through multiple pathways in Drosophila. Genes Dev 2009; 23:2333-2344.

29 Jiang H, Edgar BA. EGFR signaling regulates the proliferation of Drosophila adult midgut progenitors. Development 2009; 136:483-493.
30 Zhou F, Rasmussen A, Lee S, Agaisse H. The UPD3 cytokine couples environmental challenge and intestinal stem cell division through modulation of JAK/STAT signaling in the stem cell microenvironment. Dev Biol 2013; 373:383-393.

31 Dubreuil RR, Grushko T, Baumann O. Differential effects of a labial mutation on the development, structure, and function of stomach acid-secreting cells in Drosophila melanogaster larvae and adults. Cell Tissue Res 2001; 306:167-178.

32 Hoppler S, Bienz M. Specification of a single cell type by a Drosophila homeotic gene. Cell 1994; 76:689-702.

33 Bienz M. Endoderm induction in Drosophila: the nuclear targets of the inducing signals. Curr Opin Genet Dev 1997; 7:683-688.

34 Tanaka R, Takase Y, Kanachi M, Enomoto-Katayama R, Shirai T, Nakagoshi H. Notch-, Wingless-, and Dpp-mediated signaling pathways are required for functional specification of Drosophila midgut cells. Dev Biol 2007; 304:53-61.

35 Filshie BK, Poulson DF, Waterhouse DF. Ultrastructure of the copper-accumulating region of the Drosophila larval midgut. Tissue Cell 1971; 3:77-102.

36 Shanbhag S, Tripathi S. Epithelial ultrastructure and cellular mechanisms of acid and base transport in the Drosophila midgut. J Exp Biol 2009; 212:1731-1744.

37 Canfield V, West AB, Goldenring JR, Levenson R. Genetic ablation of parietal cells in transgenic mice: a new model for analyzing cell lineage relationships in the gastric mucosa. Proc Natl Acad Sci USA 1996; 93:2431-2435.

38 Bredemeyer AJ, Geahlen JH, Weis VG, et al. The gastric epithelial progenitor cell niche and differentiation of the zymogenic (chief) cell lineage. Dev Biol 2009; 325:211-224.

39 Bardin AJ, Perdigoto CN, Southall TD, Brand AH, Schweisguth F. Transcriptional control of stem cell maintenance in the Drosophila intestine. Development 2010; 137:705-714.

40 Nyeng P, Norgaard GA, Kobberup S, Jensen J. FGF10 signaling controls stomach morphogenesis. Dev Biol 2007; 303:295310 .

41 Sander GR, Powell BC. Expression of notch receptors and ligands in the adult gut. J Histochem Cytochem 2004; 52:509516.

42 Kim TH, Shivdasani RA. Notch signaling in stomach epithelial stem cell homeostasis. J Exp Med 2011; 208:677-688.

43 Matsuda Y, Wakamatsu Y, Kohyama J, Okano H, Fukuda K, Yasugi S. Notch signaling functions as a binary switch for the determination of glandular and luminal fates of endodermal epithelium during chicken stomach development. Development 2005; 132:2783-2793.

44 Hongyo T, Buzard GS, Palli D, et al. Mutations of the Kras and p53 genes in gastric adenocarcinomas from a highincidence region around Florence, Italy. Cancer Res 1995; 55:2665-2672.

45 Lee JW, Soung YH, Seo SH, et al. Somatic mutations of ERBB2 kinase domain in gastric, colorectal, and breast carcinomas. Clin Cancer Res 2006; 12:57-61.

46 Lee WC, Beebe K, Sudmeier L, Micchelli CA. Adenomatous polyposis coli regulates Drosophila intestinal stem cell proliferation. Development 2009; 136:2255-2264.

47 Lin G, Xu N, Xi R. Paracrine Wingless signalling controls self-renewal of Drosophila intestinal stem cells. Nature 2008; 455:1119-1123. 
48 Singh SR, Zeng X, Zheng Z, Hou SX. The adult Drosophila gastric and stomach organs are maintained by a multipotent stem cell pool at the foregut/midgut junction in the cardia (proventriculus). Cell Cycle 2011; 10:1109-1120.

49 Takashima S, Mkrtchyan M, Younossi-Hartenstein A, Merriam JR, Hartenstein V. The behaviour of Drosophila adult hindgut stem cells is controlled by Wnt and Hh signalling. Nature 2008; 454:651-655.

50 Zeng X, Chauhan C, Hou SX. Characterization of midgut stem cell- and enteroblast-specific Gal4 lines in Drosophila. Genesis 2010; 48:607-611.

51 Furriols M, Bray S. A model Notch response element detects suppressor of hairless-dependent molecular switch. Curr Biol 2001; 11:60-64.

52 Yarnitzky T, Min L, Volk T. An interplay between two EGFreceptor ligands, Vein and Spitz, is required for the formation of a subset of muscle precursors in Drosophila. Mech Dev
1998; 79:73-82.

53 Spradling AC, Stern D, Beaton A, et al. The Berkeley Drosophila Genome Project gene disruption project: single Pelement insertions mutating $25 \%$ of vital Drosophila genes. Genetics 1999; 153:135-177.

54 Lee T, Luo L. Mosaic analysis with a repressible neurotechnique cell marker for studies of gene function in neuronal morphogenesis. Neuron 1999; 22:451-461.

55 Lin $\mathrm{G}, \mathrm{Xu} \mathrm{N}, \mathrm{Xi}$ R. Paracrine unpaired signaling through the JAK/STAT pathway controls self-renewal and lineage differentiation of Drosophila intestinal stem cells. J Mol Cell Biol 2010; 2:37-49.

56 Wang C, Zhao R, Huang P, et al. APC loss-induced intestinal tumorigenesis in Drosophila: Roles of Ras in Wnt signaling activation and tumor progression. Dev Biol 2013; 378:122140 .

(Supplementary information is linked to the online version of the paper on the Cell Research website.) 